\title{
Construction of a Non-negative Solution of the Leontief-Ford Model
}

\author{
Fatima Ashakova \\ Department of Computer Science and Mathematics, U. D. Aliev Karachay-Cherkess State University, Karachayevsk, Russia \\ Email address \\ asxakova@bk.ru

\section{To cite this article:} \\ Fatima Ashakova. Construction of a Non-negative Solution of the Leontief-Ford Model. Science Journal of Circuits, Systems and Signal \\ Processing. Vol. 8, No. 1, 2019, pp. 19-23. doi: 10.11648/j.cssp.20190801.13
}

Received: June 26, 2019; Accepted: July 24 2019; Published: August 8, 2019

\begin{abstract}
The article deals with the Leontief-Ford model, which is allowed to take into account the costs that necessary for the elimination of industrial waste. We consider its non-negative solution for those cases when the errors of the initial data have little influence on the result of the solution and for those cases when the errors of the initial data significantly affect the result of the solution. In the first case, it is called correctly delivered, and in the second incorrectly delivered. We use the iteration method to find a nonnegative solution to a correctly posed model, and also we use the regularization method to find a nonnegative solution to an ill-posed model. The developed technique is brought to a practical algorithm, which is implemented in the program "LF_2", it allows you to find a solution to the model, regardless of whether the model is correctly supplied or incorrectly supplied. An example of its application is given, where we enter the initial data of a poorly conditioned balance model into the program and obtain its non-negative solution. The results of this work may be interesting for specialists in economics and mathematical methods and models, as well as economic entities. It is known from practice that in the development of balance models of economic entities there are models with a bad number of conditionality. The application of the developed software product and methods described in this paper will allow economic entities to make quality and quick management decisions on the volume of product output of each sector of the economy, taking into account the costs necessary for the elimination of industrial waste, regardless of the conditionality and dimension of their balance models.
\end{abstract}

Keywords: Leontief-Ford Model, Non-negative Solution, Regularization Method, Iteration Method

\section{Introduction}

In the production of products, there are wastes to be eliminated. Consider the Leontief-Ford model, which takes into account these circumstances.

Research of this model is devoted to a considerable number of works [1-4], which confirms its complexity and practical significance.

The considered model is called productive if it has a non-negative solution.

After determining the productivity of the model, it is necessary to determine how to find its solutions.

When applying the model to solve practical problems of large dimensions, the initial data can be given inaccurately. It is known that if small errors in the initial data do not significantly affect the result of the model solution, then the model is called correctly delivered, and if they significantly affect the result of the solution, then the model is called incorrectly delivered.

In the construction of its solution there are problems, the solution of which is devoted to this work.

If for the solution of well-posed models for high-dimensional to apply the "exact" methods, the errors of calculation are accumulated. Therefore, in this paper we will use approximate methods to solve the correctly posed model, and if the model is incorrectly set, then the regularization method.

\section{Theoretical Background}

Let's consider the model of Leontief-Ford [5-7]:

$$
\left.\begin{array}{l}
x=A_{11} x+A_{12} y+b_{1}, \\
y=A_{21} x+A_{22} y-b_{2},
\end{array}\right\}
$$

where $x$ is a vector that characterizes the gross output of the 
product (dimensions $n$ );

$y$ is a vector that characterizes industrial waste (dimensions $m$ );

$b_{1}$ is a vector that characterizes the net output of a useful product (dimensions $n$ );

$b_{2}$ is a vector, which characterizes the residual level of industrial waste (dimensions $m$ );

$A_{11}$ is a the matrix is called the matrix of direct costs (size's $n \times n)$;

$A_{12}$ is a is a matrix that characterizes the costs of elimination of industrial waste (size's $n \times m$ );

$A_{21}$ is a matrix that characterizes the volume of industrial waste (size's $m \times n$ );

$A_{22}$ is a matrix that characterizes the volume of industrial waste that appears in the process of elimination of old industrial waste (size's $m \times m$ );

$\theta$ is a zero vector (dimensions either $n$, either $m$ ).

We are only interested in non-negative solutions:

$$
x \geq \theta, \quad y \geq \theta
$$

\section{Method}

Formally, the model (1) can be written as a matrix equation:

$$
\tilde{z}=\tilde{A} \tilde{z}+\tilde{f}
$$

Where

$$
\begin{aligned}
& \tilde{A}=\left(\begin{array}{ll}
A_{11} & A_{12} \\
A_{21} & A_{22}
\end{array}\right) \\
& \tilde{f}=\operatorname{col}\left(b_{1},-b_{2}\right)^{T} \in R^{n+m}, \tilde{z}=\operatorname{col}(x, y)^{T} \in R^{n+m} \\
& \text { If }(I-\tilde{A})^{-1} \text { it exists, equation (3) can be rewritten } \\
& \tilde{z}=(I-\tilde{A})^{-1} \cdot \tilde{f}
\end{aligned}
$$

or

$$
(I-\tilde{A}) \cdot \tilde{z}=\tilde{f}
$$

here $I$ is the unit matrix.

It is known that the following conditions must be met for the model (1) to be productive

$$
\left.\rho(\tilde{A})<1, \begin{array}{c}
b_{1}+A_{11} b_{1}+A_{12} b_{2} \geq 0_{1}, \\
-b_{2}+A_{21} b_{1}+A_{22} b_{2} \geq 0_{2}
\end{array}\right\}
$$

where $\rho(\tilde{A})$ is a spectral radius of the matrix $\tilde{A}$.

$0_{1}, 0_{2}$ are null-vectors respectively of $R^{n}$ and $R^{m}$.
In this paper we consider two cases when the model (3) is correctly set, that is, if the error elements $\tilde{A}$ and elements $\tilde{f}$ models (3) lead to minor changes in the results of its solution. And the second case, when the model (3) is incorrectly set, that is, if the errors of the elements $\tilde{A}$ and elements $\tilde{f}$ models (3) lead to significant changes in the results of its decision.

In the first case, to find a solution to the model under consideration, we will use the method of successive approximations, and in the second case, the Tikhonov regularization method.

For convenience, we denote $C=(I-\tilde{A})$, then (6) takes the form:

$$
C \cdot \tilde{z}=\tilde{f}
$$

We will assume that instead of the exact values of the matrix elements $C$ and elements of the block vector $\tilde{f}$ we have their approximate values $\tilde{C}, \tilde{f}^{\bullet}$, that is.

$$
\tilde{C} \cdot \tilde{z}=\tilde{f}^{\bullet}
$$

Minor error in defining the elements of $\tilde{C}, \tilde{f}^{\bullet}$ in one case, it does not significantly affect the decision of the model $\tilde{z}$, and in another - significantly.

Denote by $\tilde{z}$ that is the solution of system (8), using $\tilde{z}^{\bullet}$ that is the solution of the system (9). The question is how different the solutions will be $\tilde{z}$ and $\tilde{z}^{\bullet}$ systems (8) and (9) under specified $\zeta$ is the absolute error of a block matrix $\tilde{C}$, that is $\|\tilde{C}-C\|<\zeta$, and $\eta$ is absolute error of block vector $\tilde{f}^{\bullet}$, that is $\left\|\tilde{f}^{\bullet}-f\right\|<\eta$. Here $\|C\|$ is the norm of the matrix C [8].

It is known [9] that a sufficiently complete information about the magnitude of the difference $\left\|\tilde{z}^{\bullet}-\tilde{z}\right\|$ can only be obtained through, matrix $C$ in (8). To do this, enter the number

$$
v(C)=\frac{\|C\|}{\left\|C^{-1}\right\|}
$$

called the condition number of the matrix $C$ (systems (8)). Here $C^{-1}$ is matrix inverse of the matrix $C$.

If $v(C)<10^{3}$, the matrix is considered to be well conditioned and the solution (8) is stable otherwise, by $v(C) \geq 10^{3}$, that is ill-conditioned and the solution (8) is unstable.

In the first case, to find a solution to the model under consideration, we will use the method of successive approximations, and in the second case, the Tikhonov regularization method. 


\section{Result and Discussion}

\subsection{Problem Statement}

In this work it is necessary to solve the following problems: to develop a method of non-negative solution of the Leontief-Ford model, for the case when it is correctly set and for the case when it is incorrectly set; to describe the algorithm of its non-negative solution; to produce a software implementation of the described algorithm and give an example of its application.

\subsection{Non-negative Solution Technique}

Consider the first case when the Leontief-Ford model is correctly supplied.

Let the model (3) have a nonnegative solution, it is unique and stable.

We construct a numerical solution of the model (3) by the method of successive approximations:

$$
\tilde{\mathrm{z}}_{\mathrm{k}+1}=\tilde{A} z_{k}+\tilde{f}, \quad \tilde{z}_{0}=\theta, \quad k=0,1,2, \ldots
$$

According to [10-12], if condition (7) is satisfied, solution (3) exists and only iterative process (11) converges at any initial approximation $\tilde{z}_{0}$. A fair assessment of the proximity

$$
\left\|\tilde{z}^{*}-\tilde{\mathrm{z}}_{\mathrm{k}+1}\right\| \leq \frac{\|\tilde{A}\|^{k}}{1-\|\tilde{A}\|}\left\|\tilde{z}_{1}-\tilde{z}_{0}\right\|
$$

where $\tilde{Z}^{*}$ is the decision (3).

Norm $\|\mathrm{A}\|$ can be specified [13]:

$$
\|\mathrm{A}\|_{1}=\max _{1 \leq j \leq n+m} \sum_{i=1}^{n+m}\left|a_{i j}\right|,\|\mathrm{A}\|_{2}=\max _{1 \leq j \leq n+m} \sum_{j=1}^{n+m}\left|a_{i j}\right|
$$

Consider the second case where the block matrix $\tilde{A}$ ill-conditioned and error $\left\|\tilde{z}^{\bullet}-\tilde{z}\right\|$ in decision $\tilde{z}$ model (3) will be significant if it is incorrectly delivered. For convenience, we will build a model solution (9). We will apply the method of regularization of A. N. Tikhonov [9, 14-16].

Suppose that

$$
\|\tilde{C}-C\| \leq \xi,\left\|\tilde{f}^{\bullet}-\tilde{f}\right\| \leq \delta
$$

From $[16,17]$ it follows that the search for a solution (9) is reduced to finding a vector $\tilde{z}^{\alpha}$, minimizing a smoothing functional:

$$
M^{\alpha}\left[\tilde{z}, \tilde{f} \tilde{f}^{\bullet} \tilde{C}\right]=\left\|\tilde{C} z-\tilde{f}^{\bullet}\right\|^{2}+\alpha \Omega[\tilde{z}], \alpha>0
$$

where $\Omega[x]=\|\tilde{z}\|^{2}$ is the stabilizing functional, $\alpha=\alpha(\delta)$ is regularization parameter. In this case, as shown in $[14,16$, 17] there is one gross output vector $\tilde{z}^{\alpha}$, which can be determined at any fixed $\alpha>0$ from the system

$$
\alpha \tilde{z}_{k}^{\alpha}+\sum_{j=1}^{n} \sum_{i=1}^{n} \tilde{c}_{i k} \tilde{c}_{i j} \tilde{z}_{j}^{\alpha}=\sum_{i=1}^{n} \tilde{c}_{i k} \tilde{f}_{i}^{\bullet}, \quad k=1,2,3, \ldots, n
$$

\subsection{Algorithm}

Based on these results, we can offer the following algorithm for constructing a solution to the problem:

1. Enter the dimension $n, m$.

2. Enter the matrix $A_{11}$, dimensions $(n \times n)$.

3. Enter the matrix $A_{12}$, dimensions $(n \times m)$.

4. Enter the matrix $A_{21}$, dimensions $(m \times n)$.

5. Enter the matrix $A_{22}$, dimensions $(m \times m)$.

6. To enter a vector $b_{1}$, dimensions $(n)$.

7. To enter a vector $b_{2}$, dimensions $(m)$.

8. Create a block matrix $\tilde{A}$.

9. Create a block vector $\tilde{f}^{\bullet}$.

10. Calculate $\tilde{C}=(I-\tilde{A})$.

11. Calculate (10), to check the condition $\tilde{C}$.

12.If $\tilde{C}$ well conditioned, then go to paragraph 13, otherwise go to paragraph 19.

13. The feasibility of the condition is checked (7).

14. Set initial approximation $\tilde{z}_{0}$.

15 . Set error $\varepsilon>0$.

16. To check the feasibility of the conditions $\|\tilde{A}\|<1$ (for the selected rate from (13)).

17.If the conditions of paragraph 16 are met, the calculations shall be made according to the formula (11) until the required error is reached $\varepsilon>0$.

18. If the condition of paragraph 16 is not met, a different rule should be chosen $\|\mathrm{A}\|$.

19. The regularization parameter is set $\alpha_{1}>0$.

20 . At the specified value $\alpha_{1}$, find a solution $\tilde{z}^{\alpha_{1}}$ systems (16).

21. At known values $\alpha_{1}, \tilde{z}^{\alpha_{1}}$, calculated value $M^{\alpha_{1}}\left[\tilde{z}^{\alpha_{1}}, \tilde{f}^{\bullet}, \tilde{B}\right]$ functional (15).

22. Specify $\alpha_{2}>0, \alpha_{2}<\alpha_{1}$.

23. At the specified value $\alpha_{2}$, find a solution $\tilde{z}^{\alpha_{2}}$ systems (16).

24. At known values $\alpha_{2}, \tilde{z}^{\alpha_{2}}$, calculated value $M^{\alpha_{2}}\left[\tilde{z}^{\alpha_{2}}, \tilde{f}^{\bullet}, \tilde{B}\right]$ functional (15).

25.If $M^{\alpha_{2}}\left[\tilde{z}^{\alpha_{2}}, \tilde{f}^{\bullet}, \tilde{B}\right]<M^{\alpha_{1}}\left[\tilde{z}^{\alpha_{1}}, \tilde{f}^{\bullet}, \tilde{B}\right]$, then proceed to the implementation of the actions specified in paragraph 27.

26.If $M^{\alpha_{2}}\left[\tilde{z}^{\alpha_{2}}, \tilde{f}^{\bullet}, \tilde{B}\right]>M^{\alpha_{1}}\left[\tilde{z}^{\alpha_{1}}, \tilde{f}^{\bullet}, \tilde{B}\right]$, that put $\tilde{z}=\tilde{z}^{\alpha_{1}}$.

27. Specify $\alpha_{3}>0, \alpha_{3}<\alpha_{2}$.

28. At the specified value $\alpha_{3}$, find a solution $\tilde{z}^{\alpha_{3}}$ systems 
(16).

29. At known values $\alpha_{3}, \tilde{z}^{\alpha_{3}}$, calculated value $M^{\alpha_{3}}\left[\tilde{z}^{\alpha_{3}}, \tilde{f}^{\bullet}, \tilde{B}\right]$ functional (15).

30.If $M^{\alpha_{3}}\left[\tilde{z}^{\alpha_{3}}, \tilde{f}^{\bullet}, \tilde{B}\right]<M^{\alpha_{2}}\left[\tilde{z}^{\alpha_{2}}, \tilde{f}^{\bullet}, \tilde{B}\right]$, then proceed to the implementation of the actions specified in paragraph 32.

31.If $M^{\alpha_{3}}\left[\tilde{z}^{\alpha_{3}}, \tilde{f}^{\bullet}, \tilde{B}\right]>M^{\alpha_{2}}\left[\tilde{z}^{\alpha_{2}}, \tilde{f}^{\bullet}, \tilde{B}\right] \quad$ that's

believing $\tilde{z}=\tilde{z}^{\alpha_{2}}$.

32. Specify $\alpha_{4}>0, \alpha_{4}<\alpha_{3}$.

33. And so on, this process continues until $(k+1)$-м step can't find $\alpha_{k+1}, \tilde{z}^{\alpha_{k+1}}$, under which $M^{\alpha_{k+1}}\left[\tilde{z}^{\alpha_{k+1}}, \tilde{f}^{\bullet}, \tilde{B}\right]>M^{\alpha_{k}}\left[\tilde{z}^{\alpha_{k}}, \tilde{f}^{\bullet}, \tilde{B}\right]$. In this case, we believe $\tilde{z}=\tilde{z}^{\alpha_{k}}$ and the calculation process will stop.

34. Display the result of the decision $\tilde{z}$.

This algorithm is implemented as a software product "L-F_2" in Delphi.

\subsection{Example}

Consider an example of its application.

If:

$$
\begin{gathered}
A_{11}=\left(\begin{array}{cccc}
0.9 & 0 & -0.4 & 0 \\
-0.7 & 0.2 & -0.02 & -0.03 \\
0.01 & 0.02 & 0.9 & -0.04 \\
0.02 & 0.01 & 0.3 & 0.82
\end{array}\right) ; A_{12}=\left(\begin{array}{c}
-0.0001 \\
-0.001 \\
-0.003 \\
-0.001
\end{array}\right) \\
A_{21}=\left(\begin{array}{llll}
-0.003 & -0.02 & 0 & 1
\end{array}\right) ; A_{22}=(-0.001) \\
b_{1}=\left(\begin{array}{llll}
56400 & 23490 & 56490 & 345200
\end{array}\right) ; b_{2}=(0)
\end{gathered}
$$

Enter the initial data of the problem in the developed program "L-F_2" get the following answer (Figure 1):

$$
\alpha=0.0005, \tilde{z}^{\alpha}=\left(\begin{array}{c}
415291,3 \\
122550,9 \\
116001,1 \\
201235,5 \\
142482,8
\end{array}\right)
$$

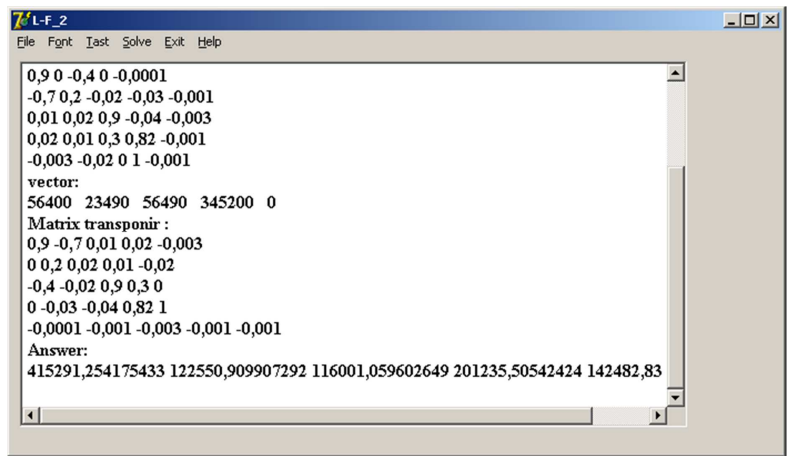

Figure 1. Program solution result «L-F_2».
According to the results of the decision, we can say that the developed program "L-F_2" allows you to find a non-negative solution to the balance model.

\section{Conclusion}

Thus, in this paper a method of non-negative solution of the model (3) is developed for the case when it is correctly set and for the case when it is incorrectly set. On the basis of this technique the algorithm of non-negative solution of the model (3) implemented in the software product is described.

The developed technique is based on the known of study results of linear algebraic equations systems by a simple iteration method, which allows to find the existence and uniqueness of a non-negative solution in the model (3) and to find out whether this solution is stable with respect to the initial conditions. It is based on the known results of the study of poorly conditioned systems of linear algebraic equations by the regularization method. Based on this technique, the algorithm of non-negative solution of the model (3) is described which allows a detailed analysis of the described model, implemented in the software product.

An example of its application is given.

The use of these software products in business entities will allow users to automate the analysis of balance sheet models and planning of gross output.

This work summarizes and complements the results of the work $[5,12,17]$.

\section{References}

[1] Potravny I., Gusev A., Stoykov V. and Gassiy V. (2017) Modification of the Leontief-Ford Input-Output Model for the Green Economy Goals and Environment Protection. Journal of Geoscience and Environment Protection, 5, pp. 15-23.

[2] Gazvan R. K., Marakhovsky A. S., Kiselyova T. V. (2017) Dual model of interindustry balance. Economy vector, no. 5 (11), pp. 20.

[3] Gulay T. A., Kopylova E. P., Surmacheva A. V. (2013) The general case of model of Leontyev - Ford. Modern high technologies, no. 6, pp. 66-67.

[4] Marakhovsky A. S. (2007) Leontiev-Ford Dynamic model with a constant level of external pollution / A. S. Marakhovsky, E. L. Toroptsev // Information systems, technologies and production management models. International scientific conf, Stavropol: SSAU, pp. 83-87.

[5] Askhakova F. H. (2016) Methods of numerical solution of the model Leontiev-Ford. World science: Problems and innovatios: collection of articles of the V International scientific-practical conference, Penza: ICNS "Science and Education", pp. 18-20.

[6] Leontief W., Ford D. (1972) Economics and mathematical methods, Moscow: Nauka, $242 \mathrm{p}$.

[7] Semenchin E. A., Askhakova F. H. (2006) Method of constructing numerical methods of solving the balance model of Leontief-Ford // Progressive technologies in learning and production: Materials of IV all-Russian conference, Kamyshin. 18-20 October 2006: 4 vol. 3, Volgograd, pp. 45-48. 
[8] Gantmacher F. R. (1966) Matrix Theory, Moscow: Science, $576 \mathrm{p}$.

[9] Numerical methods /H. S. Bakhvalov. N. P. Zhidkov, G. N. Kobelkov (2007) 5 ed., Moscow: Binom. Knowledge laboratory, $636 \mathrm{p}$.

[10] Fedoseev V. V., Garmash A. N., Dayitbegov D. M. ets. (2002) Economics mathematical methods and applied models: Studies. manual for schools, Moscow: UNITY, 391 p.

[11] Amosov A. A., Dubinsky Yu. A., Kopchenova N. V. (1994) Computational methods for engineers: Proc. benefit, Moscow: Higher. SHK, $544 \mathrm{p}$.

[12] Tovstik T. M., Volosenko K. S. (2015) Monte Carlo Algorithm for solving systems of linear algebraic equations by Seidel method. In the collection: Actual problems of computational and applied mathematics proceedings of the International conference devoted to the 90th anniversary of academician G. I. Marchuk, pp. 771-776.

[13] Semenchin E. A., Askhakova F. H. (2006) Method of constructing a non-negative solution in the model of Leontief-Ford. -In sat.: Abstracts VII all-Russian Symposium on applied and industrial mathematics, Yoshkar-Ola, pp. 347-348.

[14] Tikhonov A. N., Arsenin V. Ya. (1986) Methods of solving ill-posed problems. Textbook for universities. Ed. 3rd., corrected, Moscow: Science. Main edition of physics and mathematics literature, $288 \mathrm{p}$.

[15] Sumin M. I. (2016) Tikhonov regularization Method for solving operator equations of the first kind: Educational and methodical manual. Nizhny Novgorod: Nizhny Novgorod State University, $56 \mathrm{p}$.

[16] Verzhbitsky V. M. (2002) Fundamentals of numerical methods: Textbook for universities, Moscow: Higher school, $840 \mathrm{p}$.

[17] Askhakova F. H. (2008) Analysis of balance models of economic actors, the Karachay-Cherkess Republic, using the method of regularization. News of the Russian state pedagogical University named after A. I. Herzen, no. 36, SPb., pp. $15-17$. 